\title{
VERGLEICH DES AGONISTISCHEN VERHALTENS VON ZWEI GRUPPEN JUNGFISCHEN (HAPLOCHROMIS BURTONI) GLEICHEN ALTERS
}

\author{
von \\ A. W. H. VAN BEKKEM \& M. J. BIERHOF \\ Abteilung Tierverhalten, Universität von Amsterdam, die Niederlande ${ }^{1}$ )
}

\begin{abstract}
When a batch of young of the mouthbreeding cichlid Haplochromis burtoni is divided into two groups of equal size, they show different behaviour. The ones that are caught first are less aggressive than the second, more shy ones. If agonistic behaviour has a positive selection value, it can be an important factor for the rapid speciation in cichlid fishes. In order to investigate this assumption experimentally, one has to bear in mind that early experience, group size and group density can influence the agonistic behaviour.
\end{abstract}

\section{AUSZUG}

Eine Gruppe von Cichliden der Art Haplochromis burtoni wurde in gleiche Teile geteilt und die Aggressivität der zuerst gefangenen mit der von denen verglichen, die sich schwieriger fangen liessen. Es ergab sich, dass die scheuen Fische aggressiver waren. Wenn Aggressivität einen positiven Selektionsvorteil bietet, dann führt das dazu, einen wichtigen ursächlichen Faktor für die explosive Artbildung bei Cichliden in ihrem Angriffs- und Fluchtverhalten suchen zu müssen. Ausserdem wird darauf hingewiesen, dass die Vorgeschichte und die Gruppengrösse oder die Dichtheit in einer Gruppe für die Höhe der Aggressivität massgebend sein kann.

\section{EINLEITUNG}

Kurzes Ethogramm des Territorialverhaltens (cf. Albrecht, 1966). -

Das Versuchstier ist Haplochromis burtoni (Günther), ein maulbrütender Cichlide aus Ostafrika. Die $\sigma^{\prime} \sigma^{\circ}$ dieser Ant bilden Territorien. Die Grösse eines solchen Gebietes und die Anzahl der verschiedenen Territorien wird beeinflusst durch den Platz, der den Fischen zur Verfügung steht und dessen Gliederung durch Pflanzen, Steine usw. Jeder Fisch gründet ein Territorium, in dessen Mitte oft eine Grube im Sand gegraben wird.

Agonistische Verhaltensweisen sind beim Ein-

1) Neue Adresse: Kruislaan 322, Amsterdam-1004. nehmen und Verteidigen eines Territoriums häufig zu sehen. Doch sieht man manche dieser Verhaltensweisen auch bei Fischen ohne eigenes Gebiet und bei Jungfischen, die noch keine permanenten Territorien besetzen. Man kann die agonistische Verhaltensweisen unterscheiden in Drohen, Schwanzschlagen, Beissen, Maulzerren und Jagen. Diese Verhaltensweisen können einander abwechseln, in einander übergehen, oder auch einzeln öfter wiederholt werden:

- Drohen. Dabei stehen die Fische einander gegenüber, spreizen die Kiemendeckel und senken den Mundboden. Auch die Flossen können gespreizt werden.

- Schwanzschlagen. Mit dem Schwanz wird eine Druckwelle auf den Gegner erzeugt.

- Beissen. Dabei wird oft nur die Bewegung gemacht ohne körperlichen Kontakt.

- Maulzerren. Die Lippen des Gegners werden kurz gepackt und wieder losgelassen.

- Jagen. Der Fisch schwimmt schnell auf einen anderen zu und folgt ihm eventuell.

\section{MATERIAL UND METHODE}

Die Aquarien haben eine Grösse von $45 \times 27 \times 28$ cm ( \pm 27 l). Darin befinden sich ein kleiner Filter, ein Luftsteinchen und eine Heizung; $10 \mathrm{~cm}$ hohe Dachziegelstïcke teilen das Aquarium in drei gleich grosse Abteile.

Die Versuchstiere sind eigene Nachzucht aus dem Tierhandel. Eine Versuchsgruppe, bestehend aus zwei Würfen, zählte 90 Fische, eine zweite Gruppe, bestehend aus einem Wurf, 102 Fische. Die Nahrung besteht aus Artemien, Tetramin und Wasserflöhen.

Es wurde jeweils eine Stunde protokolliert mit einem Zehnpunktschreiber. Fünfzehn Minuten 


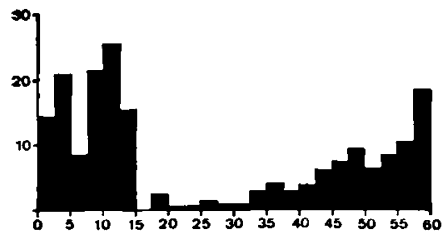

1

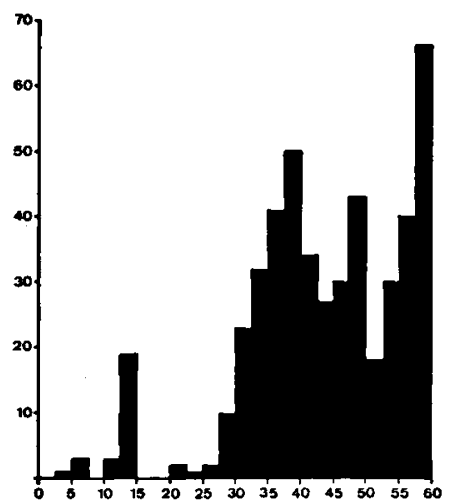

3

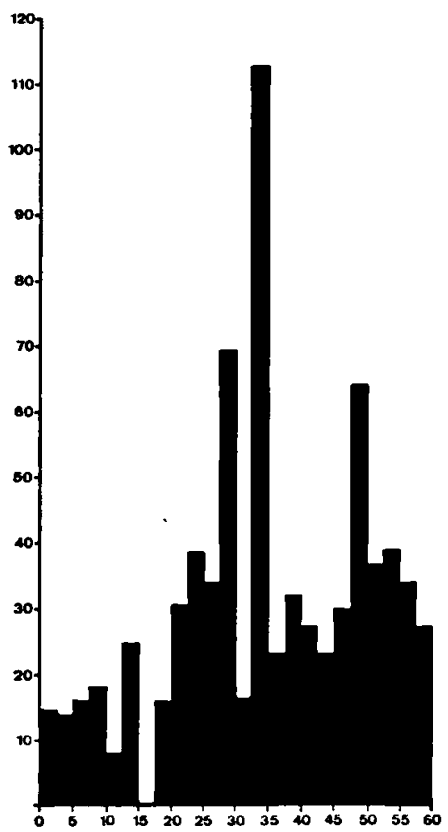

5

Histogramm 1-6. Die Abszisse gibt die Zeit in schiedenen Drohverhaltensweisen.

1 und 3: Alpha Fische,

5: Alpha-Alpha Fische,

nach Protokollbeginn wurde mit Tetramin gefüttert. Möglichst alle agonistischen Verhaltensweisen innerhalb einer Gruppe wurden registrient, da sie verhältnismässig leicht $\mathrm{zu}$ erkennen und $\mathrm{zu}$ beschreiben sind. Bei Gruppen dieser Grösse und dieses Alters war es nicht möglich, Einzeltieren $\mathrm{zu}$ folgen. Auch zum Markieren waren sie zu klein.

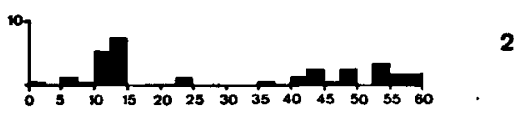

2

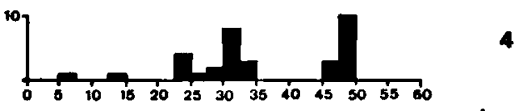

4

6

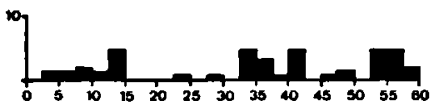

Minuten-Perioden und die Ordinate die Anzahl der ver2 und 4: Omega Fische, 6: Alpha-Omega Fische.

Es wurden 20 Protokolle gemacht, von jedem Autor 10. In gut kontrollierten Prüfprotokollen (die Beobachter konnten einander dabei weder sehen noch hören) liess sich eine hinreichende Ubereinstimmung in der Genauigkeit der Beobachter feststellen. Beim Auswerten der Protokolle wurden Verhaltensweisen, die in $2 \frac{1}{2}$ Minuten- 
Perioden vorkamen, zusammengefasst und grafisch in einem Masstab dargestellit.

Beide Gruppen wurden gespalten in je zwei gleich grosse Gruppen. Dabei wurde die Hälfte der Fische zufallsverteilt aus den Aquarium gefischt. Die zurückbleibenden Fische wurden gleich danach ebenfalls gefangen. Beide Gruppen wurden nun in möglichst gleiche aber andere Aquarien gesetzt. Die zweite Gruppe - da sie im Verlauf der Experimente die Stärkere schien - wurde Alpha Gruppe genannt und die erstgefangenen Fische Omega Gruppe. Auf diese Weise gab es zwei Alpha und zwei Omega Gruppen. Protokolle von korrespondierenden Gruppen wurden gleichzeitig gemacht. Die Alpha Gruppe von den ursprünglich 102 Fischen wurde nach Ablauf des einen Experimentes noch einmal geteilt in eine Alpha-Alpha und Alpha-Omega Gruppe. Alle Gruppen wiesen später das für diese Art übliche $\sigma^{x} \sigma^{*}:+9$ Verhältnis auf.

\section{RESULTATE}

Die erste Alpha und Omega Gruppe bestand aus je 45 Fischen. Protokolliert wurde im Alter von 39, 46, 53, 60 und 72 Tagen. Aus der Analyse der Prottokollen begaben sich die Histogramme 1 und 2. Die zweite Alpha und Omega Gruppe umfasste je 51 Fische. Das Verhalten dieser Gruppen wurde protokolliert im Alter von 60 und $67 \mathrm{Ta}$ gen. Die Analyse ergab die Histogramme 3 und 4. Nach der Teilung dieser Alpha Gruppe bestand die Alpha-Omega Gruppe aus 26 und die AlphaAlpha Gruppe aus 25 Fischen. Diese wurden protokollient im Alter von 74, 81 und 93 Tagen. Die Resultate sind aus der Darstellung Nr. 5 und 6 ersichtlich.

\section{DISKUSSION}

Für die Histogramme 1 und 2 wurden je 5 Protokolle benutzt. Es fällt auf, dass die Alpha Gruppe $\mathrm{zu}$ bestimmten Zeiten besonders aggressiv ist, und auch im allgemeinen ist die Alpha Gruppe viel aggressiver als die Omega Gruppe. Die Histogramme 3 und 4 sind gemacht aus je 2 Protokollen.
Sie zeigen dieselben Tendenzen.

Für die Alpha-Alpha und die Alpha-Omega Gruppe (Abb. 5 und 6) sind je 3 Protokolle benutzt. Die in den Abbildungen 1 bis 4 sichtbare Tendenz wird hier noch deutlicher, die Tendenz nämlich, dass zuerst gefangene Fische weniger aggressiv sind als die, die dem Fangnetz zunächst entkommen konnten.

Die Alpha-Alpha Tiere (Abb. 5) sind noch aggressiver als die Alpha Tiere (Abb. 3). Vielleicht kommt das durch das höhere Alter der AlphaAlpha Tiere; sie sind etwa einen Monat älter. Eine zweite Ursache kann darin liegen, dass die AlphaAlpha Gruppe nur halb so gross ist. Auch aus anderen Beobachtungen fiel uns auf, dass die Grösse einer Gruppe sehr wichtig ist für das Auftreten aggressiven Verhaltens. Wir teilten z.B. eine Gruppe von 30 Fischen in Gruppen von 25 und 5 Tiere. Die Zahl der Angriffe war bei der kleinen Gruppe viel höher. Schon Breder (1934), Baerends \& Baerends-van Roon (1950) und Keenleyside \& Yamamoto (1962) u.a. kamen zu ähnlichen Ergebnissen.

Aus den Resultaten wird deutlich, dass bei zufälliger Teilung einer Fischgruppe die zuerst gefangenen Fische viel wenliger aggressives Verhalten zeigen als die, die dem Fangnetz zunächst entkommen konnten. Das führt uns zu folgenden Uberlegungen: Wenn Aggressivität positiv korreliert ist mit dem Entkommen vor einem Netz oder einen Fressfeind, dann überleben und vermehren sich mehr aggressive Fische. Agonistisches Verhalten als Selektionsvorteil könnte dann auch die schnelle Artbildung innerhalb dieser Fischfamilie erklären.

Schon Worthington (1940) erwähnt die Tatsache, dass in den ostafrikanischen Seen mit Raubfischen weniger endemische Cichlidenarten vorkommen als in anderen Seen dort ohne Raubfische. In dieser Korrelation einen ursächlichen Zusammenhang zu sehen, stimmt überein mit der Annahme, dass eine raubtierfreie Umgebung adaptive Radiation begünstigt (Huxley, 1942) so wie z.B. bei den Kleidervögeln (Drepanididen) Hawais und den Galapagosfinken. Unsere Resultate brauchen dem nicht zu widersprechen. Allerdings scheint es möglich, dass ein Raubtier, wenn es das agonistische Verhalitenssystem einer Art beeinflusst, adaptive Radiation auch begünstigen kann.

\section{LITERATUR}

Albrecht, H., 1966. Zur Stammesgeschichte einiger Bewegungsweisen bei Fischen, untersucht am Verhalten von Haplochromis (Pisces, Cichlidae). Z. Tierpsychol., 23 (3) : $270-302$.
Baerends, G. P. \& J. M. Baerends-van Roon, 1950. An introduction to the study of the ethology of cichlid fishes. Behaviour, (suppl.) 1: 1-242.

BREDER, C. M., 1934. An experimental study of the repro- 
ductive habits and life history of the cichlid fish, Aequidens latifrons (Steindachner). Zoologica, N.Y., $18: 1-42$.

HUXIEY, J., 1942. Evolution, the modern synthesis: 1-645 (G. Allen \& Unwin, London).

Keenleyside, M. H. A. \& F. T. Yamamoto, 1962. Terri- torial behaviour of juvenile Atlantic Salmon (Salmo salar L.). Behaviour, 19 : 139-169.

Worthington, E. B., 1940. Geographical differentiation in fresh waters with special reference to fish. In : J. HUXLEY ed., The new systematics: $287-302$ (Oxford University Press, London etc.).

Eingegangen am 4. August 1975 